\title{
A comparison of manual and automated methods of measuring airway resistance and thoracic gas volume
}

\author{
PHILIP W. LORD AND ALAN G. F. BROOKS \\ From the MRC Environmental Hazards Unit, St Bartholomew's Hospital Medical College, \\ Charterhouse Square, London EC1M 6BQ
}

Lord, P. W. and Brooks, A. G. F. (1977). Thorax, 32, 60-66. A comparison of manual and automated methods of measuring airway resistance and thoracic gas volume. An automated method of measuring airways resistance $\left(R_{\mathrm{aw}}\right)$ and thoracic gas volume $\left(\mathrm{V}_{\mathrm{tg}}\right)$ by whole body plethysmography using a digital computer is described. The results obtained by this method are compared with those obtained by an experienced observer and the results of consistency tests on the two methods are given. There was wide variability in the values of $\mathbf{R}_{\mathbf{a w}}$ obtained using each method, and no significant differences were found between the two methods. The values of $V_{t g}$ obtained by them were highly correlated, but the results obtained manually were higher than those obtained automatically by an amount proportional to the volume. The consistency of the computer measurements was shown to be greater than that of the manual method. A significant decrease between successive measurements of $R_{a w}$ in some subjects was noted in the manual observations but not in those derived from the automated method. The new method is suitable for $\overrightarrow{\vec{O}}$ the rapid determination of $R_{a w}$ and $V_{t g}$.

The measurement of airway resistance $\left(R_{\mathrm{aw}}\right)$ and thoracic gas volume $\left(\mathrm{V}_{\mathrm{tg}}\right)$ by whole body plethysmography, as described by DuBois et al. (1956 a, b), requires the estimation of the slopes of lines obtained by displaying two signals on an oscilloscope or on an $x-y$ plotter. This estimation may be done at the time of the measurement using a cursor fitted to an oscilloscope and averaging the slopes by eye, as described by Comroe et al. (1959), or by examining the $x-y$ plot or a photograph of the oscilloscope trace. In the first of these techniques there are difficulties in estimating the slopes because they are changing rapidly, and in the other methods generally only a few slopes may be examined, and then only after some delay. We wished to examine the extent and nature of the observer error involved in using the cursor method of slope determination in order to help us to interpret the results from a long-term experiment to investigate changes in airways resistance with changes in air pollution (Lawther et al., 1973 and in preparation). We also wished to develop a fast and accurate method of measuring airways resistance, since our earlier experiments had shown that in some subjects the rise and subsequent fall of $R_{\mathrm{aw}}$ in response to the inhalation of sulphus dioxide in concentrations of more than $10 \mathrm{pp}$ could be very rapid (Lawther et al., 1975).

The new automated system is described along wi experimental work on the accuracy and reliability of both the manual and automated methods.

\section{Method}

The manual method of measuring $R_{a w}$ and followed closely that described by DuBois. After one or two minutes to allow for temperature equilibration in the plethysmograph the subjects were instructed fo make panting respirations at $2 \mathrm{~Hz}$ and with a tidal vodo ume of about $0 \cdot 2$ litre around their functional residu capacity. The average slope of the plethysmograph pressure $\left(\mathbf{P}_{\mathrm{p}}\right)$ against flow $(\dot{\mathrm{V}})$ traces between inspiratory flows of 0 to $0.41 \mathrm{sec}^{-1}$ and the average slope of the $\mathbf{P}_{\mathrm{p}}$ against mouth pressure $\left(\mathbf{P}_{\mathrm{m}}\right)$ traces, displaye on an oscilloscope, were estimated using a rotatabe cursor (Comroe et al., 1959). The slopes over 5 to 10 cycles were averaged. Thermal drift in the $P_{p}$ signal was corrected for manually by using the oscilloscowe position control. With trained subjects in the 
plethysmograph it took about 30 seconds to obtain each pair of readings.

The automated method was designed to follow the manual method closely so that they could be used independently or in parallel. The signals going to the vertical and horizontal axes of the oscilloscope were also connected to a 10 bit, \pm 1 volt FSD, analogueto-digital converter which sampled under the control of a computer program. The switching functions to seal the plethysmograph, to close the mouthpiece shutter, and to interchange the flow for mouth pressure signals were performed under program control using a digital output interface which operated relay switches. A signal line was included in the system and indicated with a lamp when the subject could start panting to obtain readings. There was a high-pass filter (cut-off at $0.05 \mathrm{~Hz} 12 \mathrm{~dB}$ octave) which was switched into the plethysmograph pressure signal line to eliminate slow drift in $\mathbf{P}_{\mathbf{p}}$. The computer used was a Hewlett-Packard 2116B. The system is shown in the block diagram (Fig. 1).

Using a simple system of commands entered through a teletype situated near the apparatus, the operator could control the course of the experiment. On being commanded to obtain readings, the computer program examined the signals (see appendix), and when values of $R_{a w}$ and $V_{t g}$ had been obtained they were immediately reported to the operator.

The analogue signals were recorded using two channels of an FM tape recorder (Hewlett-Packard 3960). A third channel was used to record dc voltage levels, generated by the program using a digital-toanalogue converter, which corresponded with the status of the program-generated switching signals. The tape was recorded at $2 \cdot 38 \mathrm{~cm} \mathrm{sec}^{-1}$.

To compare the manual and automated methods of measuring $R_{\mathrm{aw}}$ and $\mathrm{V}_{\mathrm{tg}}$ it was arranged that both the computer and an experienced operator (AB) should obtain results simultaneously from the same signals. Twelve subjects, all staff of the Environmental Hazards Unit, participated in the experiment: ages ranged from 18 to 49 years (Table) and only one (MC) was a smoker. All were healthy and had no histories of chronic respiratory illness, although one (REW) had suffered from asthma in childhood. Those who were not familiar with the plethysmographic method were given one or two practice sessions. For each subject 10 consecutive readings of $R_{a w}$ and $V_{t g}$ were obtained on five occasions. The computer was programmed to assess up to 7 slopes of each type for each of the readings.

To check the consistency of assessment by both methods the analogue signals recorded during the experiments were replayed four times, twice to $\mathrm{AB}$ and twice to the computer, and the two sets of duplicates were compared. Care was taken to maintain a constant calibration of the output signals throughout the replaying, and care was also taken to ensure that the signal gain between recording and replay was as close to unity as possible. The replayed signals were passed through two low-pass filters (cut-off $14 \mathrm{~Hz} ; 12 \mathrm{~dB}$ octave) to remove high frequency tape noise.

Owing to a fault in the recording circuitry, six of the recorded sets of 10 readings were lost, and estimated values of the missing data were calculated by the GENSTAT program employed for the analyses (Alvey, 1970).

\section{Results}

COMPARISON OF THE TWO METHODS

On two occasions the program failed to obtain readings, even though they could be obtained manually, and on two others the slopes could not be assessed manually but the program obtained readings. On this evidence the failure rate was 1 in 600 for both methods. All these four attempts were on inexperienced subjects, who for these readings produced misshapen traces.

The analyses of the results were all done in terms of resistance since a few very low readings of $R_{a w}$ gave rise to large conductances, and the distribution of the latter was markedly skew.

The relationship of the results obtained by the computer to the manually obtained readings are shown in Figs 2 and 3 against the line of identity. For $\mathrm{V}_{\mathrm{tg}}$ the regression equation of $\mathrm{V}_{\mathrm{tg}}$ (by $A B$ ) on $V_{t g}$ (by computer) had an intercept of $-0.17 \pm 0.0031$, significantly less than zero $(P<0.001)$, and had a slope of $1.075 \pm 0.007$, significantly greater than 1 (P $<0.001$ ). The relationship of $R_{a w}(A B)$ to $R_{a w}$ (computer) was very much less well defined, as expected, due to the difficulties in defining and assessing this quantity from the given signals.

Analyses of variance were performed on $\mathrm{V}_{\mathrm{tg}}$ and $\mathbf{R}_{\mathbf{a w}}$ including subject, occasion, and reading number as nested blocks and the method as a treatment. As anticipated, the sources of greatest variation were subjects and occasions. In the case of $R_{\mathbf{a w}}$ there was no difference between the two methods of assessment while for $V_{t g}$ there was a small but significant difference $(P<0.001)$, the computer readings being less than those found manually by amounts proportional to the volume. In the subject with the highest thoracic gas volume (about 6 litres), the difference was $0 \cdot 24$ litre.

In the values of $\mathbf{R}_{\mathrm{aw}}$ the most striking feature to emerge from the analysis was the decline in values between the 10 readings and the interaction of this 


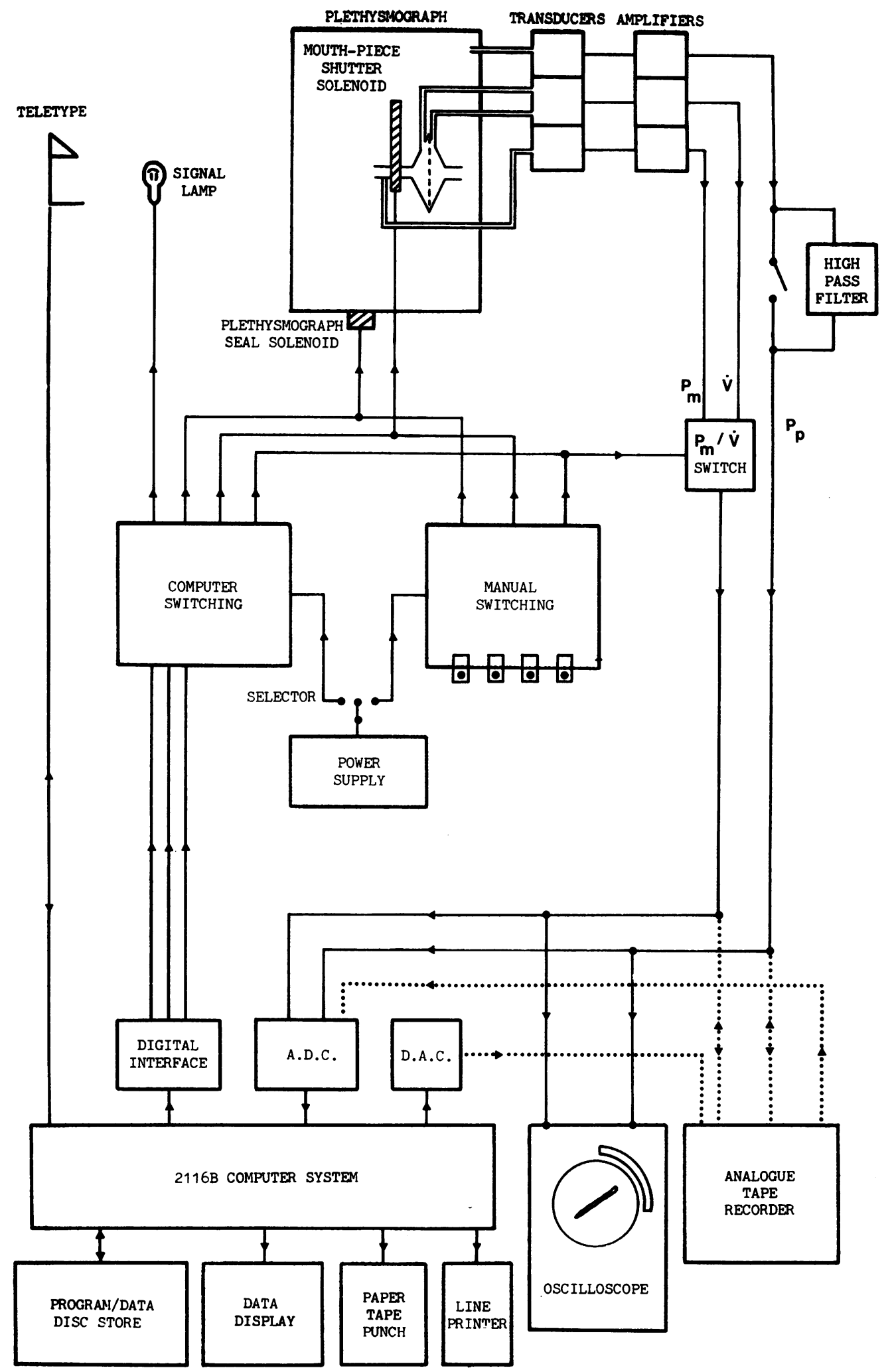

Fig. 1 Block diagram of the manual and automatic plethysmograph systems. 
Table Mean and standard errors (SE) of $R_{a w}$ and $V_{t g}$ from each method and each subject

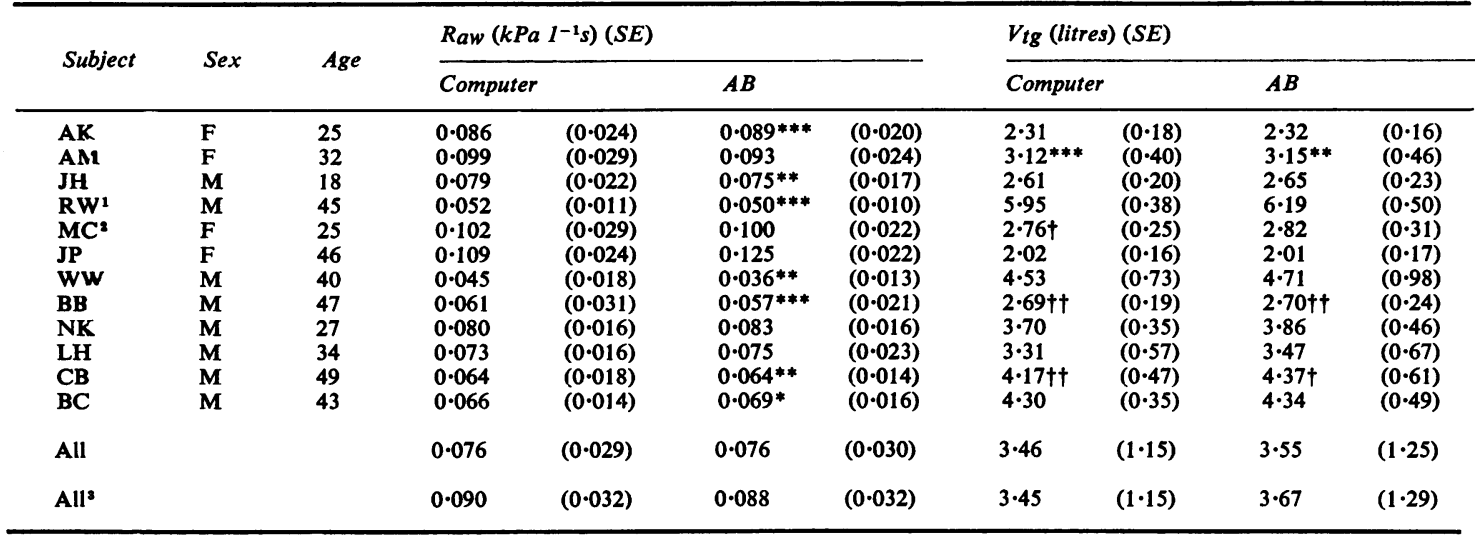

'Asthma in childhood;

${ }^{2}$ Light smoker;

${ }^{3}$ From recorded signals;

*Significant linear decrease with reading number, $\mathrm{P}<0.05$;

**Significant linear decrease with reading number, $\mathrm{P}<0.01$;

***Significant linear decrease with reading number, $P<0.001$;

tSignificant linear increase with reading number, $P<0.01$;

$+\dagger$ Significant linear increase with reading number, $P<0.001$.

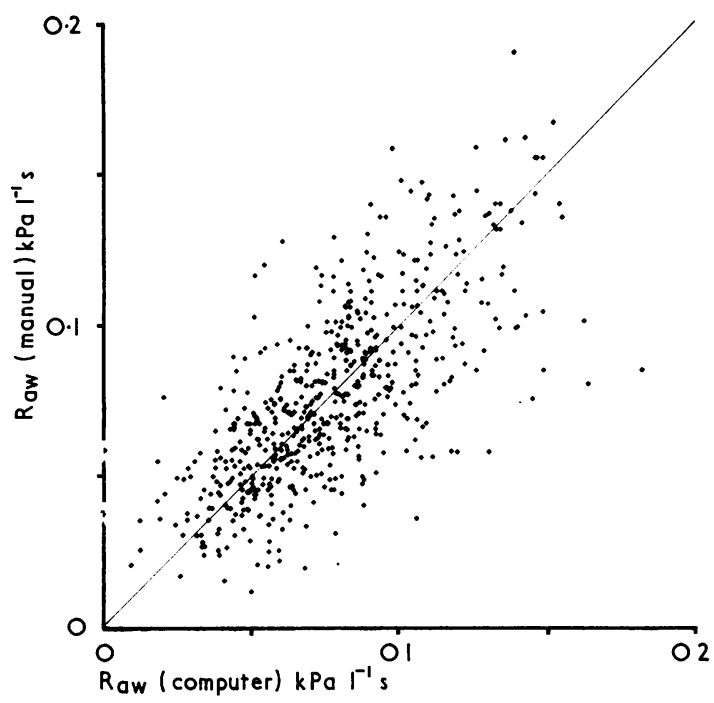

Fig. 2 Comparison of manual and automatic measurement of $R_{a w}$ against the line of identity.

trend with the method $(\mathrm{P}<0.001)$ : the trend was far more marked in the readings obtained manually than in those obtained by the computer. The analyses were repeated for each method separately, and these showed that the only significant sources of variation in the computer's readings of $\mathbf{R}_{\mathbf{a w}}$ were subjects and occasions. There was a significant linear trend in the manual readings with reading number, assigned

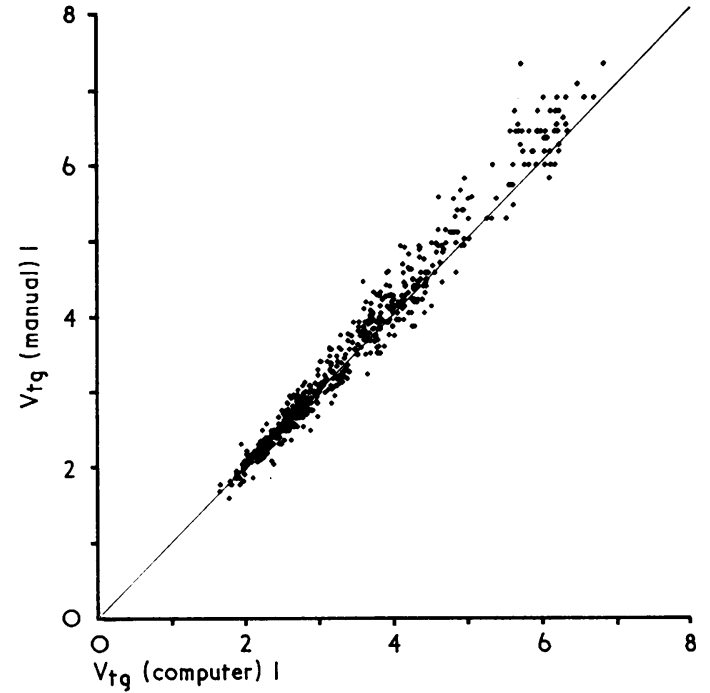

Fig. 3 Comparison of manual and automatic measurements of $V_{t g}$ against the line of identity.

values 1 to $10(\mathrm{P}<0.001)$. The interaction between this trend and the subject was significant $(P<0.01)$, indicating different trends in different subjects. To investigate this point individual analyses were performed for each subject and method separately. The results are shown in the Table; seven of the 12 subjects showed a significant linear trend with reading number in the values of $\boldsymbol{R}_{\mathbf{a w}}$ obtained 
manually. No individual trends were seen in the computer determinations. Inspection of the data showed that the readings seemed to be more uniform for readings 3 to 8 , and repeating the analysis of the manual readings taking all subjects together showed that the linear trend was then only just significant $(P<0.05)$. The means for each reading number for each method are shown in Fig. 4.

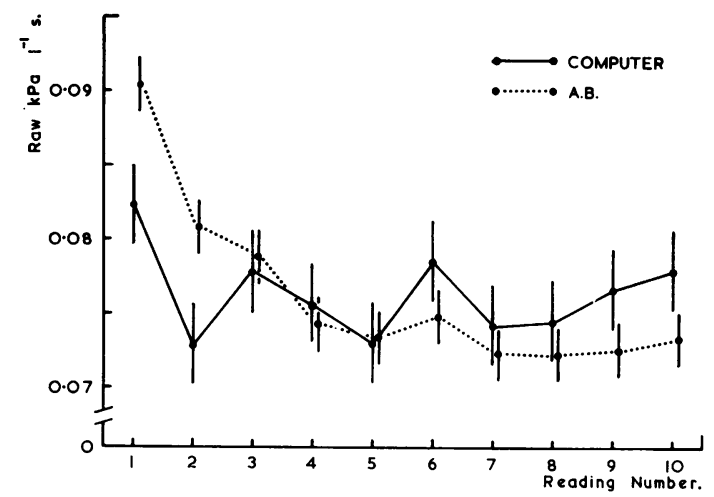

Fig. 4 Means and standard errors of Raw for each successive measurement made by the manual and automatic methods.

There was evidence from the analyses that there was a very small, but significant, linear trend with reading number for $V_{t g}$, and also a significant interaction between the trend and subjects in both methods. Analysis of $V_{t g}$ for each subject and method individually confirmed trends in some subjects and not in others (Table). Changes in $\mathrm{V}_{\mathrm{tg}}$ could have caused differences in $R_{a w}$ for these subjects, assuming conductance and $\mathrm{V}_{\mathrm{tg}}$ to be linearly related. The same analyses in terms of specific resistance rather than $R_{a w}$ gave essentially the same result, indicating that the observed changes in $R_{a w}$ were not caused by changes in $\mathrm{V}_{\mathrm{tg}}$.

\section{CONSISTENCY OF THE TWO METHODS}

The failure rate of the two methods was similar to that in the previous experiment. There were two readings of $\mathbf{R}_{\mathbf{a w}}$ and $\mathbf{V}_{\mathrm{tg}}$ which could not be determined, one for the manual method and one for the computer (where a tape fault prevented the program obtaining a reading).

The grand means of $R_{a w}$ and $V_{t g}$ from the two methods were close to those obtained in the previous series (Table). Using the first duplicate from each of the two methods, a comparison was made following the procedures described above in order to check consistency through the recording and replay process. The results were substantially the same as before.

Separate analyses were done of the compute results and those obtained by the manual method $\vec{\phi}$ treating subjects, occasions, and reading numbers as nested blocks and the duplications as a treatment? These showed no significant variation due to the $\vec{\oplus}$ duplications in $R_{\mathbf{a w}}$ or $\mathrm{V}_{\mathrm{tg}}$ in either of the methods As a measure of the relative consistency of eachs method, the percentage contribution to the sums of squares in the readings within occasion within subject stratum of the analyses due to the duplications (and their interaction with subject and reading number. were calculated. For $R_{a w}$ this gave $5.3 \%$ and $3.9 \%$ for $\mathrm{AB}$ and the computer respectively, and for $\mathrm{V}_{\mathrm{t}} \mathrm{O}$ $1.6 \%$ and $1.3 \%$ respectively. On this evidence the computer is more consistent than $\mathbf{A B}$.

\section{Discussion}

The computer method is $30 \%$ faster than the manuale method, and it could be made more rapid by inspect -7 ing fewer loops. The individual slopes of the $P_{\text {D }}$ against $\dot{V}$ signals could be used to obtain estimate of $R_{\mathrm{aw}}$, at the rate of about two per second, though their variability would be much greater than that on the median usually used to obtain readings.

The breath-to-breath changes in the slopes used to calculate $R_{a w}$ are large. In the manual methoo additional sources of error are parallax between the oscilloscope screen and the cursor and the manuab adjustment applied to compensate for drift in $\mathbf{P}_{\mathrm{p}}$ Using a subset of the recorded signals described $\vec{b}$ above, different observers have been shown to given significantly different estimates of $R_{\mathrm{aw}}$ (and $\mathrm{V}_{\mathrm{tg}}$ ) from the same signals (Lord et al., 1977). This combination of the inherent variability in the loops. defining $R_{\text {aw }}$ and the inter- and intra-observers differences make the measurement imprecise.

The significant, and systematic, difference between? the values of $V_{t g}$ obtained by the two methods could have been due to a number of causes, such as a perceptual effect, a systematic error in the scale, ov parallax error between the screen and cursor; the latter two would apply to $R_{a w}$, which shows noN systematic error.

The trend in $R_{\text {aw }}$ between the 10 successive readings observed by $A B$ (Table) is similar to trends? seen in a long series of measurements on three subjects (Lawther et al., 1973 and in preparation). The changes from the first to the tenth readings made by $\mathrm{AB}$ in these series for three subjects were $20.5 \%$ $18.6 \%$, and $15.7 \%$, which can be compared to the $18.8 \%$ seen over all subjects in the present experiment $\frac{0}{6}$ In the absence of any other satisfactory explanation 
it would seem that the effect is a perceptual one concerning the alignment of the cursor and the judgement of slopes. The different subjects produced loops which had characteristic and different shapes, and the fact that the trend was not seen in all the subjects indicates that the effect may be shape dependent, though no obvious feature of the loops could be implicated.

The trend in $V_{t g}$ with reading number was observed by each method, suggesting that the increase represents genuine changes in the levels about which some subjects chose to pant.

We would like to thank C. Brown and C. J. Derrett for help and advice with the electrical aspects of the work and Janet Edwards and Alison Macfarlane for work on the statistical analyses.

\section{Appendix}

The computer programs are written in FORTRAN IV and consist of a main program, four input, output, and control overlay segments, and a choice of two sampling segments. These two algorithms are essentially the same, an on-line version and an off-line version that uses prerecorded signals.

The range over which we usually define $R_{a w}$ is 0 to $0.41 \mathrm{sec}^{-1}$ inspiratory flow. The routines measure slopes from the $\mathrm{P}_{\mathrm{p}} v \mathrm{~F}$ signals by finding the extreme points $\left(\mathrm{P}_{1}, \dot{\mathrm{V}}_{1}\right)$ and $\left(\mathrm{P}_{2}, \dot{\mathrm{V}}_{2}\right)$, sampled within this range of the flow and calculating the slope $\left(\mathbf{P}_{2}-\mathbf{P}_{1}\right)$ / $\left(\dot{\mathrm{V}}_{1}-\dot{\mathrm{V}}_{2}\right)$. This method is found to be as satisfactory as any other more time-consuming procedure such as regression or principal axis determination. Only six or seven sampled points are obtained in the range due to limitations imposed by the hardware available, and such calculations based on so few points would not give any greater accuracy. The median, $S_{1}$, of a number of such slopes of these lines is used to calculate $R_{\mathrm{aw}}$. The median is used to exclude bias due to the occasional extreme value of a slope; the distribution of slopes has been shown to be symmetrical for both $V_{t g}$ and $R_{\text {aw }}$.

Similarly, slopes from the $P_{p} v P_{m}$ signals are determined from a line between the extreme points of a maximal range of increasing mouth pressure signal; the median, $\mathbf{S}_{2}$, of a number of slopes is used to calculate $\mathbf{R}_{\mathbf{a w}}$ and $\mathbf{V}_{\mathrm{tg}}$. The formulae used are

$$
\mathbf{R}_{\mathbf{a w}}=\mathbf{k}_{1}\left(\frac{\mathbf{S}_{1}}{\mathbf{S}_{2}}\right)-\mathrm{G}
$$

and

$$
\mathrm{V}_{\mathrm{tg}}=\mathrm{k}_{2} \mathrm{~F} \mathrm{~S}_{2}-\mathrm{D}
$$

where $k_{1}$ and $k_{2}$ are calibration constants, $G$ is the flowmeter resistance, $\mathrm{D}$ is the dead space volume of the subject and the flowmeter, and

$$
\mathrm{F}=1-\frac{\text { body volume, litres }}{605}
$$

is a correction factor for the volume of the plethysmograph occupied by the subject.

Samples of two signals $\left(\dot{V}\right.$ and $P_{p}$ or $P_{m}$ and $\left.P_{p}\right)$ are obtained every $3.5 \mathrm{msec}$. The identification of features in the traces is achieved by setting thresholds $\left(T_{1}, \ldots, T_{5}\right)$ which allow for the combined expected magnitudes of physiological and electrical noise. The

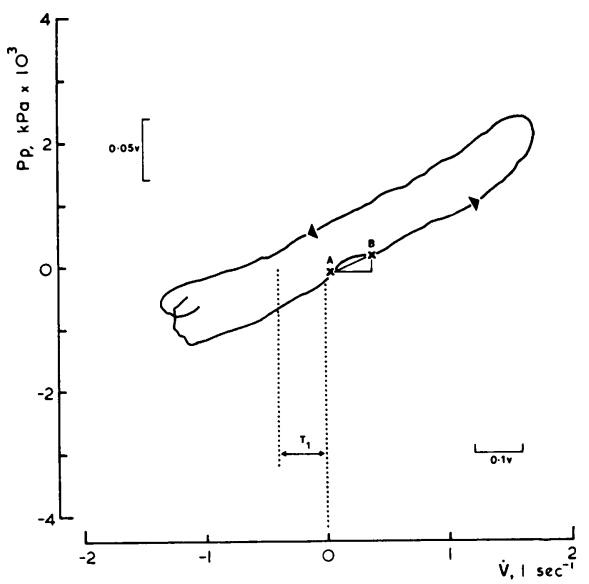

Fig. 1 Illustration of the determination of the $P_{p} \vee \dot{V}$ slopes (see Appendix).

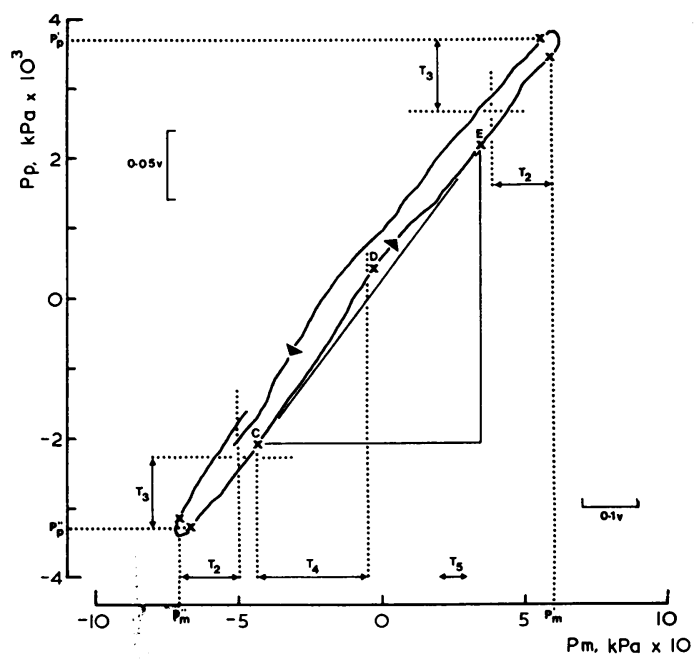

Fig. 2 Illustmation of the determination of the $P_{p} \vee P_{m}$ slopes (see Appendix). (Note that increasing $P_{m}$ signal is associated with decreasing mouth pressure.) 
algorithms for inspecting the two types of traces follow:

Errors are recorded if the time taken to complete any of the steps noted below exceeds the values indicated in brackets, and also if the total time to inspect each of the two types of traces is greater than $7 \mathrm{sec}$.

$\mathrm{P}_{\mathrm{p}} v \dot{\mathrm{V}}$ (Fig. 1)

(a) The program detects when the subject has started the panting manoeuvre by $|\dot{\mathrm{V}}|>$ $2\left|T_{1}\right|$. The plethysmograph is sealed and 1 second is allowed for $P_{p}$ to settle;

(b) expiration is sought, defined when $\dot{\mathrm{V}}<\mathrm{T}_{1}$ $(1 \mathrm{sec})$;

(c) the first sampled point which then exceeds zero flow, $\mathrm{A}$, is taken as the first point in the flow range $(0.5 \mathrm{sec}$.);

(d) sampling continues until $\dot{V}>0.41 \mathrm{sec}^{-1}$ when the previous point sampled, $B$, determines the upper end of the range. The slope of line $A B$ is calculated $(0.25 \mathrm{sec}$.);

(e) go to (b) above.

$\mathbf{P}_{\mathrm{p}} v \mathbf{P}_{\mathrm{m}}$ (Fig. 2)

(a) A maximum is sought by sampling and continuously noting the maximum values of $P_{p}$ and $\mathbf{P}_{m}\left(\mathbf{P}_{p}^{\prime}\right.$ and $\mathbf{P}_{m}^{\prime}$ respectively); a maximum is defined as having been passed when a sampled point is such that $\mathbf{P}_{\mathrm{m}}<\mathbf{P}_{\mathrm{m}}^{\prime}-\mathbf{T}_{\mathbf{2}}$ and $\mathbf{P}_{\mathrm{p}}<$ $\mathbf{P}_{\mathrm{p}}^{\prime}-\mathrm{T}_{3}(1 \mathrm{sec}$.)

(b) A minimum is sought by sampling and continuously noting the minimum readings of $P_{p}$ and $\mathbf{P}_{m}\left(\mathbf{P}^{\prime \prime}\right.$ and $\mathbf{P}^{\prime \prime}{ }_{m}$ respectively), a minimum is defined as having been passed when $\mathbf{P}_{m}>$ $\mathbf{P}^{\prime \prime}{ }_{m}+\mathbf{T}_{2}$ and $\mathbf{P}_{\mathrm{p}}>\mathbf{P}^{\prime \prime}{ }_{\mathrm{m}}+\mathbf{T}_{3}$. The first point which meets this condition, $\mathrm{C}$, is the lower end of the required line $(1 \mathrm{sec})$.

(c) A subsequent range of increasing mouth pressure signal must exceed $T_{4}$ to ensure an adequate range; the first point doing so, $D$, is noted $(0.5 \mathrm{sec})$.

(d) As in (a) above, a maximum is sought, only in doing so $P_{m}$ must not be $T_{5}$ less than it was at point $\mathrm{D}, \mathrm{P}_{\mathrm{m}}(\mathrm{D})$, for otherwise the trace is turning back on itself too soon. The point defining the upper end of the line, $E$, is then the last point sampled such that $P_{m}>P_{m}$ (D) and $\mathbf{P}_{\mathrm{m}}<\mathbf{P}_{\mathrm{m}}^{\prime}-\mathbf{T}_{2}$ and $\mathbf{P}_{\mathrm{p}}<\mathbf{P}_{\mathrm{p}}^{\prime}-\mathbf{T}_{3}$ for the new values of $P^{\prime} m$ and $P^{\prime}$. The slope of the lineo $\mathrm{CE}$ is calculated $(1 \mathrm{sec})$.

(e) Go to (b) above.

The threshold values normally used are:

$$
\begin{array}{ll}
\mathrm{T}_{1}=0.4 & 1 \mathrm{sec}^{-1} \\
\mathrm{~T}_{2}=0.2 & \mathrm{kPa} \\
\mathrm{T}_{3}=0.001 \mathrm{kPa} \\
\mathrm{T}_{4}=0.4 & \mathrm{kPa} \\
\mathrm{T}_{5}=0.1 & \mathrm{kPa}
\end{array}
$$

The end expiration condition required before closing the mouthpiece shutter is defined by first achieving $\omega_{\text {. }}$ $\dot{\mathrm{V}}<\mathrm{T}_{1}$ and then on finding $\dot{\mathrm{V}}>0$. Three seconds are ${ }_{-}$ allowed to detect this condition.

\section{References}

Alvey, N. G. (1970). GENSTAT-A General Statistical Program. Rothamstead Experimental Station, Unitedo Kingdom.

Comroe, J. H. Jr., Botelho, S. Y., and DuBois, A. B (1959). Design of a body plethysmograph for studying cardiopulmonary physiology. Journal of Applied Physiology, 14, 439-444.

Dubois, A. B., Botelho, S. Y., Bedell, G. N., Marshall, R.? and Comroe, J. H. Jr.(1956a). A rapid plethysmographics method for measuring thoracic gas volume: a com- $-\frac{\partial}{\partial}$ parison with a nitrogen washout method for measuringe functional residual capacity in normal subjects. Journag of Clinical Investigation, 35, 322-326.

Dubois, A. B., Botelho, S. Y., and Comroe, J. H. Jrö (1956b). A new method for measuring airway resistance in man using a body plethysmograph: values in norma? subjects and in patients with respiratory disease Journal of Clinical Investigation, 35, 327-335.

Lawther, P. J., Lord, P. W., Brooks, A. G. F., and Waller, R. E. (1973). Air pollution and pulmonary airway resistance: a pilot study. Environmental Research, $6_{0}^{\times}$ 424-435.

Lawther, P. J., MacFarlane, A. J., Waller, R. E., and Brooks, A. G. F. (1975). Pulmonary function an $₫$ sulphur dioxide, some preliminary findings. Environ mental Research, 10, 355-367.

Lord, P. W., Brooks, A. G. F., and Edwards, J. M (1977). Variation between observers in the estimatio of airway resistance and thoracic gas volume. Thorax 32, 68-71.

Requests for reprints to: P. W. Lord, Environment Hazards Unit, MRC Air Pollution Unit, St Bartholw mew's Hospital Medical College, Charterhouse Square London EC1M 6BQ. 